\title{
Germanica
}

allemande au XXe siècle

\section{Michael Köhlmeiers Sicht der Antike am Beispiel des Romans Kalypso}

L'image de l'antiquité dans le roman Kalypso de Michael Köhlmeiers

\section{Alfred Strasser}

\section{OpenEdition}

\section{Journals}

Édition électronique

URL : http://journals.openedition.org/germanica/2078

DOI : 10.4000/germanica.2078

ISSN : $2107-0784$

\section{Éditeur}

Université de Lille

\section{Édition imprimée}

Date de publication : 31 décembre 2002

Pagination : 175-186

ISBN : 9782913857100

ISSN : 0984-2632

\section{Référence électronique}

Alfred Strasser, « Michael Köhlmeiers Sicht der Antike am Beispiel des Romans Kalypso », Germanica [Online], 31 | 2002, Online erschienen am: 21 März 2013, abgerufen am 06 Oktober 2020. URL : http:// journals.openedition.org/germanica/2078 ; DOI : https://doi.org/10.4000/germanica.2078

Ce document a été généré automatiquement le 6 octobre 2020.

(C) Tous droits réservés 


\title{
Michael Köhlmeiers Sicht der Antike am Beispiel des Romans Kalypso
}

\author{
L’image de l'antiquité dans le roman Kalypso de Michael Köhlmeiers
}

\author{
Alfred Strasser
}

\section{I}

In ihrer Ausgabe vom 2. Februar 2002 war in der Wiener Tageszeitung « Die Presse » der Leserbrief eines gewissen Professor G.E. Schmidt ${ }^{1}$ abgedruckt, in dem er seinem «Unmut über Literatur » Ausdruck verleiht. Er meint in diesem Brief, daß sich seit einiger Zeit Autoren, Filmemacher und andere Künstler in ihrer Arbeit in Ermangelung eigener, origineller Ideen auf « die Überarbeitung bzw. Nachdichtung von Werken der Vergangenheit, die Antike eingeschlossen » beschränkten :

Was einstmals neben der Sprache, dem Ausdruck, die vornehmste Aufgabe des Verfassers war und auch heute noch sein sollte, nämlich die Konstruktion des Handlungsgefüges, die Einbettung in einen bestimmten Zeitablauf, wird von der oft recht eigenwilligen Textgestaltung rücksichtslos in den Hintergrund gedrängt. Die Handlung, die man selbst hätte erfinden sollen, wird als Aufhängevorrichtung für abstruse angeblich gegenwartsbezogene Wortkaskaden mißbraucht ${ }^{2}$.

Diese künstlerische Produktion wäre vor allem Ausdruck des "Unvermögens » zeitgenössischer Autoren, genuine Stoffe zu finden. Leider seien die als Vorlage dienenden Texte ihren Bearbeitern wehrlos ausgeliefert.

3 Sicher würde es diesem Leserbriefschreiber nicht einfallen, etwa Shakespeare, Racine, Goethe oder Hauptmann des literarischen Unvermögens zu bezichtigen, obwohl sich die eben genannten Autoren ebenfalls historischer Stoffe, auch aus der Antike, bemächtigt und sie frei bearbeitet hatten.

4 Professor G.E. Schmidt hat allerdings in einem recht: In den letzten Jahren ist eine nicht unbeträchtliche Zahl literarischer Texte, welche antike Stoffe behandeln, 
erschienen. Es sei hier nur auf Christoph Ransmayrs Ovid-Roman Die letzte Welt ${ }^{3}$ (1988), auf Sten Nadolnys Ein Gott der Frechheit ${ }^{4}$ (1994) oder auf Botho Strauß' Drama Ithaka (1998) verwiesen.

5 Geht man von der Zahl der über antike Themen publizierten Arbeiten aus, so hat sich von den zeitgenössischen Schriftstellern aber wahrscheinlich Michael Köhlmeier ${ }^{6}$ am intensivsten mit der Antike beschäftigt. Begonnen hat Köhlmeier seine Arbeit über klassische Stoffe mit einer Auftragsarbeit für den Österreichischen Rundfunk (ORF). Er sollte Gustav Schwabs Sagen des klassischen Altertums auf eine möglichst lebendige Weise neu erzählen und seine neue Fassung in einer wöchentlichen Radiosendung auch selbst vortragen.

6 Der Erfolg war so groß, daß sich der ORF entschloß, die ganze Sendereihe 1995 auf Tonträgern zu veröffentlichen; ein Jahr darauf erschien der erste Teil der Sendereihe auch als Buchausgabe ${ }^{7}, 1997^{8}$ und $1998^{9}$ folgten noch zwei weitere Teile.

7 Köhlmeier ließ es damit allerdings nicht bewenden. Die Antike übte auf ihn eine derartige Anziehungskraft aus, daß er sich nicht auf die Nacherzählung von Mythen und Heldenliedern beschränken konnte : «Wenn man anfängt, sich mit der Antike zu beschäftigen, besteht die Gefahr, daß man süchtig wird ${ }^{10}$, meinte er und begann mit der Arbeit an einer Romantrilogie. Sein Interesse galt dem Vater der klassischen Literatur überhaupt, nämlich Homer, und besonders seinem zentralen Helden Odysseus: Die Odyssee sollte die Grundlage seiner geplanten Romantrilogie werden. Köhlmeier steht damit am Ende einer langen und reichen Tradition von Bearbeitungen des Odyssee-Stoffes, die in der Antike begonnen hat und über die Jahrhunderte bis zur Gegenwart reicht ${ }^{11}$.

8 Als erster Teil erschien 1995 der Roman Telemach ${ }^{12}$. Darin ist der Themenkreis der Telemachie behandelt, also vor allem die vier ersten Gesänge der Odyssee. Der zweite Roman erschien 1997 unter dem Titel Kalypso ${ }^{13}$ und hat Odysseus' siebenjährigen Aufenthalt bei der gleichnamigen Nymphe zum Inhalt. Ein abschließender Teil sollte von der Heimkehr des Odysseus nach Ithaka handeln, doch dieser ist bis heute noch nicht erschienen.

9 Angesichts der thematischen Komplexität der beiden Romane, die es sicherlich wert wären, daß man sich ausführlich damit beschäftigt, soll in diesem beschränkten Rahmen nur die Analyse von Kalypso Gegenstand der Untersuchung sein. Der Roman wurde in dem inzwischen eingestellten Fernsehliteraturspektakel «Literarisches Quartett » von zwei der Diskutanten als « Kitsch » abgetan, darüber hinaus warf man dem Autor noch vor, er sei "unfähig", der heutigen Welt mit antiken Geschichten beizukommen ${ }^{14}$. Zwar hat auch diese negative Besprechung die Verkaufszahlen des Romans in die Höhe getrieben, doch vielleicht war die mediale «Exekution » für den literarisch gewöhnlich sehr fruchtbaren Köhlmeier der Grund, von einer Veröffentlichung des letzten Teils der Trilogie abzusehen. Diese Annahme muß aber im Bereich der Spekulation bleiben, die wirklichen Gründe sind nicht bekannt.

10 Jene Fernsehkritik darf auf keinen Fall ein Hindernis für die Beschäftigung mit dem Roman sein. Die Sendung « Literarisches Quartett » war als nichts Anderes zu sehen als der mediale Ersatz antiker Gladiatorenkämpfe, wobei es dem Hauptmoderator vor allem darum ging, in ähnlich selbstherrlicher Weise wie die Cäsaren Schriftstellerköpfe rollen zu lassen. Diese Sendung war aber keinesfalls der Ort seriöser Literaturkritik. 
11 In Homers Epos Odyssee ist die Zeit, die Odysseus bei Kalypso verbringt, sehr kurz abgehandelt. In kaum 200 Versen erzählt der Dichter Odysseus' sieben Jahre lang dauernden Aufenthalt bei der Nymphe. Erzählzeit und erzählte Zeit stehen hier in einem krassen Mißverhältnis, ist doch diese Station in Odysseus' zehnjähriger Irrfahrt von allen seinen Erlebnissen die längste. Im Epos dagegen nimmt sie den Status einer fast nebensächlichen Episode ein, viele andere Begebenheiten erscheinen dem Verfasser weit wichtiger. Doch vergegenwärtigen wir uns vorerst das Geschehen in Homers Epos :

Nachdem Odysseus Schiffbruch erlitten hat, bei dem alle seine Gefährten ums Leben gekommen sind, treibt er neun Tage lang im Meer umher, bis er in der darauffolgenden Nacht auf die Insel Ogygia angeschwemmt wird. Herrin dieser Insel ist die Nymphe Kalypso. Sie nimmt Odysseus bei sich auf und verliebt sich in ihn. In ihrer Zaubergrotte erleben beide den Rausch der sinnlichen Liebe. Um immer in diesem Zustand der Glückseligkeit zu verbleiben, will Kalypso Odysseus für ewig an sich binden und ist dafür bereit, ihm Unsterblichkeit zu verleihen. So leben Odysseus und Kalypso sieben Jahre lang allein auf der Insel, doch Odysseus wird auf Ogygia nicht glücklich, er muß ständig an seine Heimat und seine Familie denken.

Als nun der ihm feindlich gesinnte Meeresgott Poseidon, welcher für sein Herumirren auf den Meeren verantwortlich ist, bei einem Fest der weit entfernt wohnenden Äthopier weilt, beschließt Zeus, auf Initiative Athenes, Odysseus' Irrfahrt ein Ende zu bereiten und ihm die Heimkehr zu den Seinen zu erlauben. Der Götterbote Hermes wird damit beauftragt, den Entschluß aus dem Olymp Kalypso mitzuteilen. Sie vernimmt entrüstet die Botschaft der Götter, erklärt sich aber schließlich bereit, Odysseus ziehen zu lassen.

$$
\begin{aligned}
& \text { Doch zu Odysseus ging, dem großgesinnten, die hehre } \\
& \text { Nymphe, als sie des Zeus Gebote vernommen, und fand ihn, } \\
& \text { Der am Ufer des Meeres saß; ihm wurden die Augen } \\
& \text { Nicht mehr trocken von Tränen; das süße Leben zerrann ihm; } \\
& \text { Der sich nach Heimkehr sehnte ; denn nicht mehr gefiel ihm die } \\
& \text { Nymphe ; } \\
& \text { Sondern die Nächte wohnte er noch erzwungen der Göttin, } \\
& \text { Selbst nicht wollend der wollenden, bei in der wölbigen Grotte } \text {; }^{15}
\end{aligned}
$$

14 Kalypso erkennt, daß Odysseus schon lange nicht mehr freiwillig bei ihr ist, seine Gedanken sind bei den Seinen in Ithaka. Nicht einmal das nächtliche Liebesspiel kann ihm die Sehnsucht nach seiner Heimat nehmen.

Odysseus baut also ein Floß, Kalypso besorgt ihm für die Überfahrt Kleider und versorgt ihn mit Mehl, Wasser und Wein. Und nachdem die Nymphe Odysseus geschworen hat, ihm keinen Schaden zufügen zu wollen, verabschiedet er sich von ihr und sticht in See.

Die Kürze der Darstellung dieser langen Zeitspanne ruft beim Leser von Homers Epos naturgemäß eine Reihe von Fragen hervor : Warum wird die Zeit zwischen Odysseus' Ankunft auf Ogygia und seiner Abreise im Epos fast gänzlich ausgespart? Was hat Odysseus bei der Nymphe sieben Jahre lang getan bzw. haben die beiden auch noch etwas anderes getan als einander zu lieben?

Michael Köhlmeier versucht auf diese Fragen zu antworten und durch seinen Roman die im Epos verbliebenen leeren Stellen zu füllen. Er siedelt die antike Handlung in der 
Gegenwart an, verzichtet dabei aber weder auf die vielen Figuren des antiken Vorbilds noch auf den Olymp mit allen seinen Göttern.

Die Berührung von Gestalten der Antike mit der Gegenwart führt zu einer Reihe von Anachronismen, die sich durch den ganzen Roman hindurchziehen. Kalypso telefoniert ganz selbstverständlich mit ihrem Handy, fährt mit dem Bus ins Einkaufszentrum, kauft dort für Odysseus ein Heft der Comic-Reihe Calvin und Hobbes, und als sie einmal in der Stadt einen One-Night-Stand erleben will, geht sie mit ihrem Auserwählten ins «Oriental », Wiens Luxusstundenhotel von Weltruf. Andrerseits greift der Erzähler gerne zu Vergleichen mit bekannten Schauspielern oder Sängern der Gegenwart, um neu eingeführte Figuren zu charakterisieren.

Vom Leser werden keine Kenntnisse der klassischen Texte vorausgesetzt. Ganz nach dem Vorbild seines antiken Musters charakterisiert der Erzähler, der gerne von sich in der etwas antiquierten Wir-Form spricht, wortreich jede neu eingeführte Figur, versieht sie mit einer oder mehreren Eigenschaften, die leitmotivisch bei der Erwähnung der Figur immer wieder genannt werden. Er stellt Verbindungen zwischen den Figuren her und erzählt ihre Geschichte, sofern es für das Verständnis der Handlung nötig ist. Dabei folgt er Homer, nimmt sich aber auch die Freiheit, Figuren zu erfinden, wenn das für den Handlungsverlauf oder die Schlüssigkeit der Erzählung notwendig ist.

\section{III}

Am Anfang des Hauptteils von Köhlmeiers Roman - der Beginn der eigentlichen Kalypso-Handlung - stellt der Erzähler fest, daß Odysseus während seiner Irrfahrt zwar viele Abenteuer $\mathrm{zu}$ bestehen hatte, jedoch habe er die längste Zeit nicht mit Abenteuern und Kämpfen zugebracht, sondern sei in der Gesellschaft von Frauen gewesen : Kalypso war die eine, Kirke die andere. Auch bei Kirke blieb Odysseus über ein Jahr lang und zeugte sogar mit ihr einen Sohn.

Odysseus, der nach der heiligen Troja Zerstörung [...] so vieler Menschen Städte gesehen und Sitte gelernt hat und auf dem Meere so viel Leiden erduldet, seine Seele zu retten und seiner Freunde Zurückkunft ; der von den zehn Jahren Irrfahrt, wie wir leicht aus den Geschichten errechnen können, knapp neun sich bei Frauen aufhielt $; 16$

21 Diese Tatsache steht in krassem Gegensatz zu dem in der Odyssee erzählten Geschehen. Die heroische Männerwelt Homers verbietet jedoch die detaillierte Darstellung jeder Mann-Frau-Beziehung. Der Held wird nicht im Bett einer Frau zum Helden, sondern sein Ruhm ist das Ergebnis seiner Fähigkeit, Gefahren die Stirn zu bieten und Konflikte unter Anwendung von List oder Gewalt zu lösen.

Heute ist eine solche Art von Heldentum fragwürdig geworden, ja sie gehört der Vergangenheit an. Die Bezeichnung « Held » im Sinne der Antike flößt der Welt dem so Bezeichneten gegenüber nicht mehr schaurige Verehrung ein, sie ist vielmehr Synonym einer archaischen Methode der Konfliktlösung und eine andere Bezeichnung für Mörder. So gibt Odysseus' Jugendliebe Aphaia, der er in der Unterwelt begegnet, diesem zu verstehen, daß sich für sie und die Gesellschaft Helden überlebt haben und von niemandem mehr gebraucht werden : 
Ein Held bist du geworden? Was ist ein Held? Eine zum Aufrechtgehen dressierte Bestie? Was soll ich damit? Wer soll damit etwas anfangen? Wen interessiert das! 17

Das Augenmerk des Erzählers wendet sich also naturgemäß von der Perspektive des antiken Autors ab und findet als Ersatz dafür das Thema der sinnlichen Leidenschaft, verkörpert durch die Nymphe Kalypso.

In ihr sieht der Erzähler das Idealbild der Frau als erotisches Wesen: Sie hat ideale Körpermaße, volle, aber nicht zu volle Lippen von schwerer roter Farbe und goldbrauens, zu Flechten gebundenes Haar. In ihrem Anwesen lebt sie alleine in Harmonie mit der Natur, immer nackt und zieht Kleider nur dann an, wenn sie mit dem Bus in die Stadt fährt, entweder um Einkäufe zu machen oder um nach Männern Ausschau zu halten, die es aber wegen ihrer vollkommenen Schönheit nicht wagen, sie anzusprechen.

Als Kalypso den an den Strand der Insel Ogygia angeschwemmten, entkräfteten und geschundenen Odysseus entdeckt, bringt sie ihn zu sich nach Hause, pflegt ihn hingebungsvoll, füttert ihn und salbt seinen Körper solange, bis er wieder zu Kräften kommt. In der Folge entwickelt sich zwischen ihr und Odysseus eine Beziehung, die ausschließlich auf physischem Begehren und der Erlangung höchster körperlicher Lust beruht :

Er blickte auf Kalypsos wunderbaren Rücken nieder; auf ihren hell ihm entgegenblühenden Arsch, sah die allen Kurven vorbildliche Linie von der Hüfte zur Taille, sah ihre makellose Haut, dankte nach allen Seiten des Universums, daß dieser Frau eine solche Haut gegeben worden war, damit sie vor ihm darin prunkte. Es ist Ficken, sagte er sich, es ist bloß Ficken und nicht Gottesdienst, man kann es durchaus nachlässig betreiben ${ }^{18}$.

Während Odysseus, bei aller körperlichen Befriedigung, diesem Treiben gegenüber Distanz wahrt (es ist nicht Gottesdienst, also nicht unmittelbar wichtig), trachtet Kalypso danach, die Momente höchster Ekstase in eine Dimension der Ewigkeit zu führen. Solches Trachten nach Ewigkeit ist jedem Lustgewinn ihhärent, denn « Lust will aller Dinge Ewigkeit, will tiefe, tiefe Ewigkeit! $»^{19}$ Kalypso meint, diese ewige Lust erreichen zu können, indem sie Odysseus zu einem Unsterblichen macht :

Kalypso, die Nymphe, war unsterblich und sie konnte unsterblich machen, den sie liebte. Genauer müßte es heißen: Ihr Zustand war unvergänglich, und in diese Unvergänglichkeit konnte sie heben, wen sie liebte. Bisher war ihr niemand begegnet, an dem sie diese Macht beweisen mochte ${ }^{20}$.

Die im Olymp versammelten Götter wollen sie dabei unterstützen; sie möchten dem Phänomen der Unsterblichkeit auf den Grund gehen und veranstalten zu diesem Zweck, natürlich nur für Unsterbliche, ein Symposion über das Thema, wobei Odysseus das Objekt ihrer Forschungen sein soll. Pallas Athene und Merkur werden nach einer ersten Sitzung als Beobachter zu Kalypso und Odysseus geschickt; sie sollen, getarnt in den Comic-Figuren Calvin und Hobbes die beiden beobachten und regelmäßig dem Olymp berichten.

Trotz seiner intensiven, leidenschaftlichen Beziehung zu Kalypso, lehnt Odysseus ihr Angebot ab, er verzichtet auf ewiges Leben und damit auf eine Existenz der Leidenschaft und Ekstase. Er entfremdet sich zunehmends der Nymphe, immer öfter und immer länger sucht er die Einsamkeit des Strandes, blickt lange aufs offene Meer, in die Richtung in der er sein geliebtes Ithaka vermutet. 
Es mag befremdlich erscheinen, daß Odysseus das Angebot der Unsterblichkeit nicht annimmt, weil er, kurz bevor er auf Ogygia angespült wird, den Hades betreten hat und weiß, was ihn nach dem Tod erwartet. Er kennt die Misere der Sterblichkeit, das Schicksal der Toten, die verdammt sind, im Reich der Unterwelt ein Schattendasein zu führen.

Bei Homer sucht Odysseus, auf Anraten Kirkes, den Hades auf, um den thebanischen Seher Tiresias, der auch nach dem Tod seine seherischen Fähigkeiten nicht verloren hat, nach der Zukunft und besonders nach dem besten Heimweg nach Ithaka zu befragen. Am Rande der Welt angekommen, opfern Odysseus und seine Gefährten den Göttern Schafe und lassen das Blut der Tiere in eine Grube fließen. Sogleich erscheinen die Schatten aus dem Totenreich und wollen von diesem Blut trinken, um so für kurze Zeit wieder ins Leben zurückzukehren. Odysseus verwehrt ihnen dies, solange Tiresias nicht getrunken und seine Weissagung gemacht hat.

Nach dem Treffen mit dem Seher begegnet er seiner Mutter Antikleia, die, aus Gram über Odysseus Schicksal, gestorben ist und ihm jetzt über das Befinden der Seinen zu Hause auf Ithaka berichtet. Schließlich ziehen die früheren Gefährten vom trojanischen Feldzug an ihm vorbei, der ermordete Agamemnon und Achilleus, der Odysseus eindringlich vor dem Grauen der Unterwelt warnt :

'Tröste mich nicht, Odysseus, strahlender, über den Tod weg.

Lieber wollte ich als Tagelöhner den Acker bestellen

Bei einem armen Mann, der nicht viel hat an Besitztum,

Als über alle die Toten, die hingeschwunden, herrschen ${ }^{21}$.

Es erscheinen noch Tantalos, Sisyphos, Tityos und viele andere, bevor Odysseus diesen grauenvollen Ort verläßt und auf seinem Schiff das Weite sucht.

Im Gegensatz zur Odyssee, wo die Nekyia etwa in der Mitte des Epos beschrieben wird, beginnt Köhlmeier seinen Roman mit Odysseus' Gang in den Hades. Auch hier trifft er auf die Schatten einer Reihe berühmter Toter ; sie bilden aber nur den Rahmen für die Begegnung mit seiner Jugendliebe Aphaia. Köhlmeier erfindet jene Figur und dieser dramaturgische Trick ermöglicht eine Rückblende auf Odysseus' Jugend. Ein »unheroischer» Lebensabschnitt kann so beleuchtet und damit das aus dem antiken Text bekannte Gesamtbild korrigiert und ins richtige Licht gesetzt werden.

In Odysseus' Jugendjahren war Aphaia die Schönheit des Strandes. Sie stand stets im Mittelpunkt einer Gruppe von Jungen, doch Odysseus gehörte nicht zu ihnen. Obwohl er sie anhimmelte, und sie um seine Gefühle wußte, beachtete sie ihn wegen seines jungen Alters nicht. Er selbst war sehr schüchtern und wagte es nie, sie anzusprechen. Eines Tages stürzte sich Aphaia, sechzehnjährig, von einem Felsen am Strand von Aigina ins Meer und starb. Ihre Leiche wurde nie gefunden.

5 Jetzt, in der Unterwelt, trifft Odysseus wieder auf seine Jugendliebe. Sie, die ihn zu Lebzeiten ständig übersehen hat, fleht ihn nun an, er solle versuchen, so wie einst Orpheus seine Euridice, sie aus dem Schattenreich zu befreien. Doch Odysseus denkt nur daran, mit Aphaia zu schlafen, und dieser Gedanke lähmt ihn in einem Maße, daß er unfähig ist, einen Rettungsversuch zu unternehmen: Er vertut die Chance und Aphaia wird von der Unterwelt wieder aufgesogen.

36 Als Odysseus also auf Ogygia strandet, hat Eros Besitz von ihm ergriffen. Er bringt die besten Voraussetzungen mit sich, um mit Kalypso eine Existenz der permanenten Ekstase zu führen. Was veranlaßt ihn nun aber, auf den ununterbrochenen Orgasmus zu verzichten? Warum sitzt er, krank vor Heimweh, am Strand und blickt wehmütig in 
die Ferne? Was bewegt ihn, anstatt eines ewigen Rausches der Sinnenfreuden die Existenz eines Sterblichen führen zu wollen, mit all seinen Unzulänglichkeiten und Nöten, die unweigerlich im Reich der Schatten enden wird?

Ein erster Zweifel über die Sinnhaftigkeit ewigen Lebens kommt Odysseus bei dem Gedanken an Tithonos ${ }^{22}$, dem zwar die Götter Unsterblichkeit, nicht aber ewige Jugend verliehen haben. Tithonos muß deshalb erleben, daß er mit zunehmenden Alter immer unansehnlicher wird, sein Körper langsam immer stärker verfällt, ohne daß er die Möglichkeit hat, diesen Verfall aufzuhalten, nicht einmal durch den Tod.

So wurde der schöne Tithonos in Ewigkeit jeden Tag älter [...] die Haut an seinen Ellbogen wurde fühllos und schrumpelig, die Tränensäcke schwollen an, seine Zähne wurden stumpf und gelb, sein Atem roch nach Kot, sein süßer Hintern wurde schlaff, die Därme quollen auf und hoben den Leib, seine weiß schimmernde Haut wurde trüb käsig und häßliche braune Flecken breiteten sich darauf aus, der Speichelfluß des Mundes ließ sich nicht mehr halten, und die Augen schwammen in ihren Höhlen wie Sardellen im öl23.

Doch über diesen verhängnisvollen Irrtum der Götter hinaus existieren für Odysseus andere, weit schwerwiegendere Gründe, um die Unsterblichkeit abzulehnen, Gründe, die Kalypso als Unsterbliche nicht verstehen kann. Sie lebt ausschließlich in der Gegenwart, sie existiert « im Reich der Wiederholung, der süchtigen Wiederholung [...] der Lust [...]. Kalypsos Märchen [...] ist ewige Wiederkehr des Immer-Gleichen ${ }^{24}$, während Odysseus als Sterblicher auf den Tod hin lebt. Alles, was er tut und erlebt, ist begrenzt, seine ganze Existenz steht im Zeichen von Chronos' Herrschaft.

Die unsterblichen Götter können das Gefühl für Zeit jedoch nicht haben. Ein Augenblick kann für sie "nach menschlichem Maß eine Nanosekunde ebenso sein wie eine Dreiviertel Woche $»^{25}$ wie der Erzähler leitmotivisch ständig wiederholt.

Unsterblichkeit ist zudem auch mit dem Verlust einer anderen Kategorie verbunden, die die menschliche Existenz ausmacht und die erst durch ein zeitlich begrenztes Dasein erlebbar wird, nämlich das Erlebnis von Glück und Unglück. Glück ist genauso wie Zeit eine Eigenheit der menschlichen Existenz. Das Wissen um dieses Erleben und die Besinnung auf den glücklichsten und unglücklichsten Moment in seinem bisherigen Dasein bringen Odysseus endgültig dazu, auf eine göttliche Existenz zu verzichten. Weder der glücklichste noch der unglücklichste Moment seiner Existenz stehen im Zusammenhang mit dem orgiastischen Treiben bei Kalypso, das « bloß Ficken » war.

41 Sowohl der Augenblick seines höchsten Glücks als auch der des bittersten Unglücks liegen weit zurück, in der Zeit, bevor Odysseus in den Krieg gezogen ist. Damals, im Alter von fünfundzwanzig Jahren, bewirtschaftete er gemeinsam mit seiner Frau Penelope ein Landgut. Er lebte zufrieden mit seiner Frau vom Ertrag seiner Ländereien und wollte eine Zeitung gründen. In diese Zeit fiel die Geburt seines Sohnes Telemach, dessen Kommen für Odysseus das höchste Glück bedeutete.

Der unglücklichsten Tag in Odysseus' Existenz ist jener Tag, an dem ihm dieses kleinbürgerliche Glück genommen wird, als ihn die Boten des Menelaos auffordern, seinen Verpflichtungen als Partner in einem militärischen Bündnis nachzukommen und mit dem alliierten Heer Helena aus der Hand der Trojaner zu befreien. Odysseus steht innerlich vor der Wahl zwischen privatem Glück und der Erfüllung seiner öffentlichen Verpflichtung. Er entscheidet sich für das familiäre Glück und will der Pflicht entgehen, indem er vorgibt, verrückt zu sein. Doch seine Verstellung wird von Palamedes durchschaut und so bleibt Odysseus keine andere Wahl, als seine Familie 
und sein Landgut zu verlassen und in den Krieg zu ziehen. Der Abschied von Penelope und Telemach ist aber weit mehr als nur die Trennung von dem zufriedenen Dasein in der Familie. Es ist der Ausgangspunkt einer tiefgreifenden Veränderung im Wesen des Odysseus, hin zum Heros, zum unerbittlich mordenden Soldaten, als welcher er schließlich berühmt werden wird.

Die Sehnsucht nach der Wiederherstellung des verlorengegangenen Familienglücks bestimmt immer häufiger seine Tage auf Ogygia, und als Kalypso wieder einmal ihren Wunsch ausspricht, für immer mit Odysseus vereint zu sein, eröffnet er ihr, daß er nur an die Heimkehr zu seiner Familie denke, bei der alleine er glücklich war.

'Daß du immer bei mir bleibst, das will ich', sagte sie, die spendend und verschwendend ist. 'Wenn du bei mir bleibst, wirst du nicht sterben, und niemand wird dich zwischen den Fingern zerreiben wie ein Minzeblatt, und du wirst nicht endlos altern wie der arme Tithonos.' - 'Aber ich vermisse meine Frau zu Hause in Ithaka', sagte er. 'Ich will zu ihr, zu ihr will ich und zu meinem Sohn.' - 'Ist sie schöner als ich ?' - 'Nein.' - 'Kommt es ihr besser als mir ?' - 'Nein.' - 'Warum willst du dann zu ihr ?' - 'Ich vermisse sie. Und ich vermisse meinen Sohn. Wir waren eine Familie. Die Familie war der glücklichste Augenblick meines Lebens' ${ }^{26}$. 
seiner Vorstellung von Glück. Das Dasein als « Kriegsheld » oder als « Frauenheld » sind nur zwei verschiedene Seiten der Entfremdung vom eigentlichen Glück : einem Leben als Teil einer Familie. In Köhlmeiers Roman ist Odysseus also das, was er, nach Horkheimer und Adorno, seit der Organisierung der klassischen Mythen durch Homer geworden ist : das « Urbild des bürgerlichen Individuums $»^{28}$.

\section{NOTES}

1. Aus dem Absender des Leserbriefes geht nicht hervor, ob es sich um einen Mann oder um eine Frau handelt.

2. G.E. Schmidt, Unmut über Literatur, in : Die Presse, 2. Februar 2002, Spectrum, p. X.

3. Chrisoph Ransmayr, Die letzte Welt, Greno, Nördlingen, 1988.

4. Sten Nadolny, Ein Gott der Frechheit, Piper, München, Zürich, 1994.

5. Botho Strauß : Ithaka, dtv, München, 1998.

6. Michael Köhlmeier wurde 1949 in Vorarlberg geboren, verbrachte seine Kindheit in Hohenems (Vorarlberg) und bei einer Tante in Deutschland. Nach der Matura studierte er von 1970 bis 1976 Politologie und Germanistik in Marburg sowie Philosophie und Mathematik in Gießen. Während des Studiums trat er gemeinsam mit Reinhold Bilgeri als Liedermacher in Erscheinung. Nach dem Studium arbeitete er mehrere Jahre lang für den Österreichischen Rundfunk, bevor er sich 1985 als freier Schriftsteller in Hohenems niederließ. Er ist Träger zahlreicher Literaturpreise und einer der fruchtbarsten österreichischen Autoren der Gegenwart.

7. Michael Köhlmeier, Michael Köhlmeiers Sagen des klassischen Altertums, Piper, München, Zürich, 1996.

8. Michael Köhlmeier, Michael Köhlmeiers neue Sagen des klassischen Altertums von Eos bis Aeneas, München, Zürich, Piper 1997.

9. Michael Köhlmeier : Michael Köhlmeiers neue Sagen des klassischen Altertums von Amor und Psyche bis Poseidon, Piper, München, Zürich, 1998.

10. Stefanie Schild, Nullnummer des Abendlandes, in : Münchner Merkur, 18./19.11.1995.

11. Von den übrigen Bearbeitungen der Odyssee seien hier nur die wichtigsten genannt: Kratinos : Odysseus (Komödie 500/420 v. Chr.) ; Gabriel Pereira de Castro (1571-1632) : Ulyssea ou Lysboa edificada (1636) ; Simon Rettenbacher (1634-1706) : Ulysses (Drama, 1680) ; Ludvig Holberg (1684-1754) : Ulysses von Ithaka (Drama, 1723/24) ; Alfred Tennysson (1809-1892) : Ulysses (Gedicht, 1833) ; Paul Heyse (1830-1914) : Odysseus (1872) ; Gerhart Hauptmann (1862-1946) : Der Bogen des Odysseus (Drama, 1914); James Joyce (1882-1941) : Ulysses (Roman, 1922); Benjamin Fondane (1898-1944) : Ulysse (Gedicht, 1933); Nikos Kasantsakis (1882-1957): Odysséos/Odysseia (Epos, 1938); Kurt Klinger $\left({ }^{*} 1928\right)$ : Odysseus muß wieder reisen (Drama, 1954).

12. Michael Köhlmeier, Telemach, Piper, München, Zürich, 1995.

13. Michael Köhlmeier, Kalypso, Piper, München, Zürich, 1997.

14. Siehe dazu : Wespennest 111, /1998, p. 53-56.

15. Homer, Odyssee, Übersetzt von Roland Hampe, Reclam, Stuttgart, 1979, p. 78.

16. Op. cit., 12, p. 47.

17. Ibid., p. 19.

18. Ibid., p. 118. 
19. Friedrich Nietzsche : Also sprach Zarathustra, hrsg. von Giorgo Colli und Mazzino Montinari, kritische Studienausgabe Bd. 4, dtv/de Gruyter, München, Berlin, New York, 1988, p. 403.

20. Op. cit. 12, p. 55.

21. Op. cit. 15, p. 187.

22. Tithonos war der ältere Bruder des Priamus und legendärer König von Troja. Er fiel vor allem durch seine ganz besondere Schönheit auf, weswegen Eos, die Göttin der Morgenröte, auf ihn aufmerksam wurde und ihn schließlich entführte. Aus ihrer Verbindung mit Tithonos gingen zwei Söhne hervor: Memnon und Emathion. Als Eos bemerkte, daß sie zwar ewig jugendlich blieb, ihr Mann allerdings alterte, erbat sie von Zeus Unsterblichkeit für Tithonos, was ihr der Göttervater auch gewährte. Leider hatte sie aber vergessen auch ewige Jugend für ihren Mann zu erbitten. So wurde Tithonos zwar unsterblich aber der Alterungsprozeß wurde nicht aufgehalten. Er wurde immer unansehnlicher, bis Eos ihn in eine Zikade verwandelte.

23. Op. cit. 12, p. 127.

24. Ibid., p. 165.

25. Ibid., p. 175.

26. Ibid., p. 323.

27. Ibid., p. 435.

28. Max Horkheimer, Theodor W. Adorno, Dialektik der Aufklärung. Philosophische Fragmente, Fischer, Frankfurt/Main 1995, p. 50.

\section{RÉSUMÉS}

In seinem Roman Kalypso erzählt der österreichische Schriftsteller Michael Köhlmeier die in Homers Odyssee nur etwa zweihundert Verse lange Episode von Odysseus' Aufenthalt bei Kalypso neu. Köhlmeier interessiert sich dabei besonders für die Ereignisse, die Homer nicht beschreibt, nämlich das Zusammenleben von Odysseus und Kalypso und das Erleben höchster sinnlicher Lust. Kalypso will, daß der Rausch mit Odysseus ewig dauern soll und bietet deshalb Odysseus die Unsterblichkeit an. Dieser lehnt aber ab, denn ein Leben in ständiger sinnlicher Ekstase, genauso wie die Existenz als Kriegsheld, bedeuten die Entfremdung vom eigentlichen Glück, dem Leben mit der Familie. Am Ende verläßt er, der Prototyp der bürgerlichen Existenz, mit dem Einverständnis der Götter Kalypso und bricht zu den Seinen auf.

Dans le roman Kalypso, l'écrivain autrichien Michael Köhlmeier réécrit les deux cents vers de l' Odyssée qui relatent le séjour d'Ulysse auprès de Calypso. Ce qui intéresse surtout Köhlmeier, c'est la réalité qu'Homère ne décrit pas : la vie commune d'Ulysse et de Calypso, leur plaisir charnel. Calypso voudrait faire durer éternellement cet enivrement des sens, c'est pourquoi elle offre à Ulysse l'immortalité. Mais celui-ci refuse, car pour lui une vie réduite à l'ivresse des sens ou à l'héroïsme du guerrier reviendrait à se détourner du véritable bonheur qu'est la vie de famille. À la fin du roman, Ulysse, l'archétype de la vie bourgeoise, quitte Calypso avec l'assentiment des dieux et rejoint les siens. 
AUTEUR

ALFRED STRASSER

Université Charles-de-Gaulle-Lille 3 